\title{
Xanthophyll Carotenoids Reduce the Dysfunction of Dermal Fibroblasts to Reconstruct the Dermal Matrix Damaged by Carbonylated Proteins
}

\author{
Yumiko Yamawaki ${ }^{1,2 *}$, Taeko Mizutani ${ }^{1}$, Yuri Okano ${ }^{1}$, and Hitoshi Masaki ${ }^{2}$ \\ ${ }^{1}$ CIEL CO., LTD., 5-4-30 Nishihashimoto, Midori-ku, Sagamihara-shi, Kanagawa 252-0131, JAPAN \\ ${ }^{2}$ School of Bioscience and Biotechnology, Tokyo University of Technology, 1404-1 Katakura-machi, Hachioji, Tokyo 192-0982, JAPAN
}

\begin{abstract}
Although extracellular carbonylated proteins (CPs) are found at higher levels in sun-exposed skin, their impact on the cellular functions of fibroblasts and their involvement in the progression of photoaging skin are not fully clarified. In our previous study, we reported that extracellular CPs increase levels of intracellular oxidative stress and result in the accumulation of newly synthesized CPs in normal human dermal fibroblasts (NHDF). Furthermore, fibroblasts exposed to CP-BSA, which is a model of extracellular CPs, had upregulated expression levels of mRNAs encoding matrix metalloproteinase-1 (MMP-1) and interleukin-8/CXCL8 (IL-8/CXCL8). These facts suggested the possibility that extracellular CPs induce a fragile structure in the dermis through the degradation of collagen and elastin. The purpose of this study was to characterize the efficacy of natural carotenoids, such as astaxanthin analogs, produced by Hematococus pluvialis (CHPs) to improve the impaired functions of fibroblasts exposed to CPs. CHPs suppressed the intracellular CP levels elevated by CP-BSA, restored mRNA expression levels of factors involved in the formation and assembly of collagen and elastin fibers and improved the formation of those fibers impaired by CP-BSA. We conclude that CHPs function as antiaging substances due to their restoration of the impaired formation of collagen and elastin fibers caused by extracellular soluble CPs.
\end{abstract}

Key words: carbonylated protein, IL-8/CXCL8, MMP-1, carotenoids

\section{Introduction}

Environmental stimuli strongly influence skin conditions because the skin is located at the outermost layer of the human body. Among those stimuli, sunlight is the most powerful influence. Sunlight, particularly excessive ultraviolet(UV) light, causes sunburn in the skin as an acute reaction following the induction of skin pigmentation. Furthermore, chronic sun exposure accelerates the appearance of aging symptoms in the skin due to structural and biochemical alterations of the dermis. One of the biochemical alterations that occurs in the dermal matrix is the presence of carbonylated proteins (CPs) at higher levels in the dermis at chronically sun-exposed sites ${ }^{1)}$.

CPs, one type of oxidized proteins, are synthesized by reactions between reactive aldehyde compounds (RACs) yielded during lipid peroxidation and the amino groups of lysine and arginine residues in proteins. Because CPs have a yellow brown color, the shift in skin color to yellow-dark with aging has been explained as the accumulation of CPs in the dermis ${ }^{1,2)}$. In addition, we have reported that the accumulation of CPs around skin pores makes them appear dark and leads to an uneven skin color ${ }^{3)}$. In addition to the optical impact of CPs on skin appearance, it has been known that CPs in corneocytes are correlated with the loss of skin moisture functions ${ }^{4}$. Furthermore, we demonstrated that CPs become inducers of oxidative stress under exposure to sunlight. CPs generate superoxide anion radicals $\left(\cdot \mathrm{O}_{2}{ }^{-}\right)$from molecular oxygen by one electron transfers through type I photosensitizing reactions caused by the absorption of blue light ${ }^{5}$. These facts suggest that CPs construct an oxidation loop in the skin under exposure to sunlight due to their generation of $\cdot \mathrm{O}_{2}{ }^{-}$. However, although the influence of CPs on skin appearance and the chemical features of CPs are understood, their impacts on cellular functions are not fully understood.

On the other hand, we have reported the possibility that CPs influence the cellular functions of fibroblasts to regulate the intracellular redox balance and to regenerate the

*Correspondence to: Yumiko Yamawaki, CIEL CO., LTD., 5-4-30 Nishihashimoto, Midori-ku, Sagamihara-shi, Kanagawa 252-0131, JAPAN

E-mail: y.yamawaki@ciel-tokyo.com

Accepted January 19, 2021 (received for review July 14, 2020)

Journal of Oleo Science ISSN 1345-8957 print / ISSN 1347-3352 online

http://www.jstage.jst.go.jp/browse/jos/ http://mc.manusriptcentral.com/jjocs 
dermal matrix ${ }^{6)}$. Extracellular soluble CPs increase intracellular CP levels though the generation of reactive oxygen species (ROS), and concomitantly alter cell shape to be enlarged and flattened. Furthermore, exposure of fibroblasts to extracellular soluble CPs upregulates mRNA expression levels of matrix metalloproteinase-1 (MMP-1) and interleukin-8/CXCL8 (IL-8/CXCL8) and shifts the balance of the metabolism of the dermal matrix towards degradation. These results indicate that conditions where CPs are present in higher amounts induce the depletion of fibrillar proteins such as collagen and elastin by influencing the functions of fibroblasts.

Meanwhile, it has been reported that acrolein, which is an $\alpha, \beta$-unsaturated aldehyde, is a major aldehyde in the stratum corneum that synthesizes $\mathrm{CPs}^{7,8)}$. In our study, carbonylated BSA (CP-BSA), which is a model of extracellular soluble CPs, caused the generation of acrolein in fibroblasts and increased levels of CPs. These facts suggest that scavenging acrolein would be an effective approach to reduce extracellular CPs in addition to reducing ROS. In our previous study, we reported that carotenoids including $\beta$-carotene scavenged acrolein ${ }^{6)}$. These facts suggest that carotenoids would suppress the dysfunction of fibroblasts induced by extracellular soluble CPs on regeneration of the dermal matrix.

Hematococcus pluvialis (HP), which belongs to the Haematococcaceae family, is a species of freshwater green algae. One interesting feature of HP is that xanthophyll carotenoids containing astaxanthin analogs are synthesized in large amounts. In fact, our preliminary analysis found astaxanthin esters, $\beta$-carotene and lutein, in the lipid fraction of HP. Those results suggested that the lipid fraction of HP could be effective in eliminating the adverse phenomena induced by extracellular soluble CPs on the cellular functions of fibroblasts.

Thus, the study was conducted to evaluate the potential of the lipid fraction of $\mathrm{HP}(\mathrm{CHP})$ to regenerate the dermal matrix focusing on the formation of collagen and elastin fibers.

\section{Materials and Methods}

\subsection{Reagents}

Xanthophyll carotenoids from Hematococcus pluvialis (CHP) were a kind gift of Kuehnle AgroSystems, Inc. (HI, USA). Bovine Serum Albumin (BSA) and the BCA protein assay kit were obtained from Sigma-Aldrich (MO, USA). 4-hydrazino-7-nitrobenzofurazan hydrazine (NBD-H) and acrolein monomer were obtained from Tokyo Chemical Industry(Tokyo, Japan). Acrolein RED was obtained from Funakoshi Co., Ltd. (Tokyo, Japan). The type I collagen antibody was obtained from Rockland Immunochemicals (PA, USA). The fibrillin-1 antibody, Hoechst 33342 and flu-
orescein-5-thiosemicarbazide(FTSC) were obtained from Invitrogen (CA, USA) . Anti-rabbit $\operatorname{Ig} G(\mathrm{H}+\mathrm{L}), \mathrm{F}\left(\mathrm{ab}^{\prime}\right) 2$ Fragment (Alexa Fluor ${ }^{\circledR} 594$ Conjugate) and anti-mouse IgG $(\mathrm{H}+\mathrm{L}), \mathrm{F}\left(\mathrm{ab}{ }^{\prime}\right) 2$ Fragment (Alexa Fluor ${ }^{\circledR} 488$ Conjugate) were obtained from Cell Signaling Technology (MA, USA). The anti-human MMP-1 antibody and the IL-8/CXCL8 ELISA kit were purchased from R\&D Systems (MN, USA).

\subsection{Components of xanthophyll carotenoids from HP}

Lipids from Hematococcus pluvialis (HP) (CHP)were prepared by extraction of lyophilized algae with ethanol. CHP contained $2.2 \%$ of carotenoids. The identification and the quantification of carotenoids in CHP was carried out by Eurofins Craft Technologies (NC, USA) with HPLC. The HPLC analysis identified components as astaxanthins $(73 \%)$, lutein $(16 \%)$ and $\beta$-carotene $(11 \%)$. In addition, the compositions of astaxanthins were $76 \%$ monoesters and $24 \%$ diesters.

\subsection{Synthesis of CP-BSA by oxidation of BSA with acro- lein}

To oxidize BSA to CP-BSA, 50 mg BSA was incubated with $150 \mathrm{mM}$ acrolein in an aqueous solution for 2 days at $37^{\circ} \mathrm{C}$. CP-BSA was purified by dialysis in MEMBRA-CEL ${ }^{\circledR}$ dialysis tubing, MWCO 3500 (Fisher Scientific, MA, USA). Protein contents were quantified using a BCA protein assay kit. The degree of carbonylation was determined using a regression equation prepared by utilizing the reaction between acrolein and NBD-hydrazine. After dialysis, the carbonylation degree in CP-BSA was determined as 25 $\mu$ mole acrolein per mg protein. The CP-BSA was used for following examinations at a concentration of $250 \mu \mathrm{g} / \mathrm{mL}$.

\subsection{Scavenging against acrolein}

A mixture of $1 \mathrm{mM}$ acrolein aqueous solution and CHP at various concentrations prepared with DMF in equivalent volumes was incubated at $37^{\circ} \mathrm{C}$ for 2 h. $250 \mu \mathrm{M} \mathrm{NBD}-\mathrm{H}$ was incubated with the reaction mixture in $0.05 \%$ trifluoroacetic acid at $37^{\circ} \mathrm{C}$ for $30 \mathrm{~min}$. The fluorescence intensity (F.I.) of the solution (Ex: 470 nm, Em: 550 nm) was then measured using a microplate reader (Spark 10M, TECAN, Männedorf, Switzerland). The scavenging ratio is expressed as a percentage as shown in the following equation:

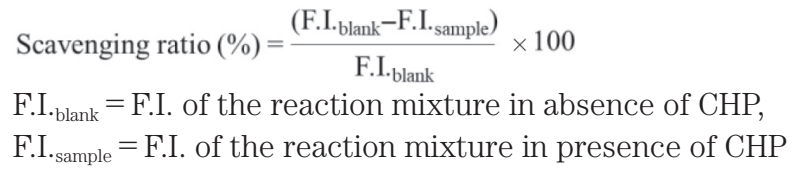

\subsection{Cell culture}

Normal human dermal fibroblasts (NHDFs) (Kurabo, Osaka, Japan)were cultured in Dulbecco's modified Eagle medium (DMEM) (Nissui, Tokyo, Japan), supplemented with $5 \%$ fetal bovine serum (FBS) (Biowest, Nuaillé, France) and incubated in a humidified atmosphere of $95 \%$ 
air and $5 \% \mathrm{CO}_{2}$ at $37^{\circ} \mathrm{C}$. NHDFs were cultured with CP-BSA or BSA in the presence or absence of CHP, and 24 $\mathrm{h}$ later, cells were harvested and re-inoculated into culture dishes.

\subsection{Detection of intracellular acrolein and CPs in NHDFs}

Intracellular acrolein in NHDFs was visualized by cultivating NHDFs in the presence of Acrolein RED $(20 \mu \mathrm{g} / \mathrm{mL})^{9)}$ at $1 \mathrm{~h}$ after treatment with BSA or CP-BSA. NHDFs treated with BSA or CP-BSA for $24 \mathrm{~h}$ were further cultured for 24 $\mathrm{h}$ after re-inoculation. and then intracellular CPs were fluorescence-labeled with FTSC and nuclei were stained with Hoechst 33342 after fixation with cold methanol. Fluorescence images originating from acrolein and CPs were obtained using a fluorescence microscope BZ-X800 (Keyence, Osaka, Japan) or a confocal laser scanning microscope (Olympus Corp., Tokyo, Japan).

\subsection{Quantitative real-time PCR (qRT-PCR) analysis}

NHDFs were further cultured for $24 \mathrm{~h}$ after re-inoculation. Total RNAs were extracted from the cells and mRNA expression levels were characterized using the real-time PCR method. Reverse transcription was performed using a GeneAmp PCR system (Applied Biosystems, USA) with ReverTraAce qPCR RT Master Mix (Toyobo, Japan). qRT-PCR was performed using a StepOne ${ }^{\mathrm{TM}}$ real-time PCR system with Power SYBR Green Master Mix (Applied Biosystems, USA). The primers used were from Takara Bio (Japan). mRNA expression levels of the following markers were examined; COL1A1 (type I collagen), MMP-1, MRC2/Endo180 (mannose receptor C type 2), FBN-1 (fibrillin-1), ELN (tropoelastin), LOX (lysyl oxidase) and IL-8/CXCL8. Glyceraldehyde 3-phosphate dehydrogenase (GAPDH) was used as a housekeeping gene. The primer sets used were as follows: COL1A1 forward, 5'-CCCGGGTTTCAGAGACAACTTC-3', and reverse, 5'-TCCACATGCTTTATTCCAGCAATC-3', MMP-1 forward, 5'-AAGGTGGACCAAC A A T T T C A GA - $3^{\prime}$, a n d reverse, 5' TGAAGGTGTAGCTAGGGTACATCAA-3', MRC2/Endo180 forward, 5'-GCTTGCACCAACATCACCAT-3', and reverse, 5'-GGGAGAAGCTGCTCTGCTCA-3', FBN-1 forward, 5'CTTCCACCTAACAGGCCATTAACA-3', and reverse, 5'CTATCACATGGTTCCATAGGTGCAG-3', ELN forward, 5'GCTGACGCTGCTGCAGCCTA-3', and reverse, 5' CAGCAAAAGCTCCACCTACA-3', LOX forward, 5'TTCTTACCCAGCCGACCAAGATA-3', and reverse, 5'GTGTTGGCATCAAGCAGGTCA-3', IL-8/CXCL8 forward, 5'-ACACTGCGCCAACACAGAAATTA-3', and reverse, 5'TTTGCTTGAAGTTTCACTGGCATC-3', GAPDH forward, 5'-GCACCGTCAAGGCTGAGAAC-3', and reverse, 5'-TGGTGAAGACGCCAGTGGA-3'.

\subsection{Quantification of MMP-1 and IL-8/CXCL8}

NHDFs were further cultured for $24 \mathrm{~h}$ after re-inocula- tion. Culture media (CM) of NHDFs collected after the further $24 \mathrm{~h}$ cultivation were used for the quantification of MMP-1 and IL-8/CXCL8. MMP-1 protein levels in the CM were analyzed by Western blotting (WB). IL-8/CXCL8 levels in the CM were quantitated using an ELISA kit.

\subsection{Visualization of collagen and elastin fibers}

NHDFs were cultured with CP-BSA or BSA in the presence or absence of CHP for 1 week. After fixation with a $4 \%$ paraformaldehyde phosphate buffer solution for 15 min at room temperature, collagen fibers and microfibrils, which are the structural fibers of elastin fibers, were immunostained with the following procedure. NHDFs were incubated with antibodies against type I collagen(1:500) or fibrillin-1 (1:100) at $4^{\circ} \mathrm{C}$ overnight. After washing, cells were incubated with anti-rabbit IgG conjugated Alexa Fluor ${ }^{\circledast} 594$ (for collagen, 1:1000) or anti-mouse IgG conjugated Alexa Fluor ${ }^{\circledR}$ 488 (for fibrillin-1, 1:1000). Nuclei were stained with Hoechst 33342. Fluorescence images were obtained with a fluorescence microscope BZ-X800 (Keyence, Japan) or a confocal laser scanning microscope(Olympus Corp., Japan).

\subsection{Statistics}

All data are expressed as means $\pm \mathrm{SD}$. Comparisons between two groups were performed using Student's t-test. A $p$-value of less than 0.05 is considered statistically significant.

\section{Results}

\subsection{Scavenging property of CHP against acrolein and the reducing effect of $\mathrm{CHP}$ on $\mathrm{CP}$ accumulation}

Treatment with CHP reduced acrolein levels during a $2 \mathrm{~h}$ incubation in a dose-dependent manner (Table 1). Interestingly, NHDFs treated with CP-BSA showed the generation of acrolein intracellularly (Fig. 1a). This result suggests that levels of intracellular CPs in NHDFs would be increased by cultivation in the presence of CP-BSA. To determine whether NHDFs treated with CP-BSA would have increased intracellular levels of CPs by physical adsorption at the cell surface, cells were re-inoculated to measure the level of intracellular CPs. NHDFs treated with CP-BSA had a higher intensity of fluorescence, which is derived from CPs, compared with cells treated with BSA (Fig. 1b).

On the other hand, NHDFs treated with CP-BSA in the presence of various concentrations of CHP had decreased intensities of fluorescence in a dose-dependent manner. Those results indicated that CP-BSA, i.e. extracellular soluble CPs, enhanced levels of intracellular oxidative stress and that treatment with CHP reduced the intracellular levels of oxidative stress elevated by CP-BSA. 
Table 1 Scavenging property of CHP against acrolein.

\begin{tabular}{cc}
\hline CHP $(\mathrm{mg} / \mathrm{ml})$ & Scavenging ratio (\%) \\
\hline 0.00 & $0.00 \pm 1.02$ \\
0.05 & $3.91 \pm 0.40$ \\
0.09 & $10.02 \pm 1.26$ \\
0.19 & $22.35 \pm 1.27$ \\
0.38 & $40.79 \pm 1.10$ \\
0.75 & $57.18 \pm 0.68$ \\
\hline
\end{tabular}

Acrolein and CHP at several concentrations were incubated in an aqueous solution for $2 \mathrm{~h}$ at $37^{\circ} \mathrm{C}$. Acrolein levels were determined with NBD-H in $0.05 \%$ trifluoroacetic acid by measuring the fluorescence intensity (F.I.) (Ex: $470 \mathrm{~nm}$, Em: $550 \mathrm{~nm}$ ). The scavenging ratio is expressed as a percentage as shown in the following equation;

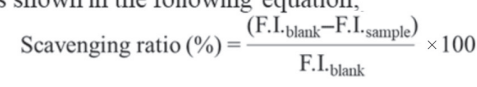

F.I.blank $=$ F.I. of the reaction mixture in absence of CHP,

F.I. sample $=$ F.I. of the reaction mixture in presence of CHP

\subsection{Amelioration effects of CHP on dermal matrix related proteins altered by CP-BSA}

Treatment with CP-BSA altered mRNA expression levels of proteins involved in the metabolism of the dermal matrix as follows; COL1A1, MRC2, FBN-1, ELN and LOX were downregulated while MMP-1 and IL-8/CXCL8 were upregulated (Fig. 2). Those results suggested that CP-BSA could interfere with the normal metabolism of the dermal matrix. On the other hand, treatment with CHP ameliorated the mRNA expression levels of COL1A1, MMP-1, MRC2/ Endo180, FBN-1 and ELN that were altered by CP-BSA. We then examined the effects of CHP on the expression of those proteins focusing on MMP-1 and IL-8/CXCL8, whose mRNAs were significantly upregulated by CP-BSA. Treatment with CP-BSA increased protein levels of MMP-1 and IL-8/CXCL8, which is consistent with the upregulation of their mRNA expression levels, but treatment with CHP obviously reduced the increased levels of both proteins that were elevated by CP-BSA (Fig. 3).

Data are presented as means \pm standard deviation (S.D.).

a)
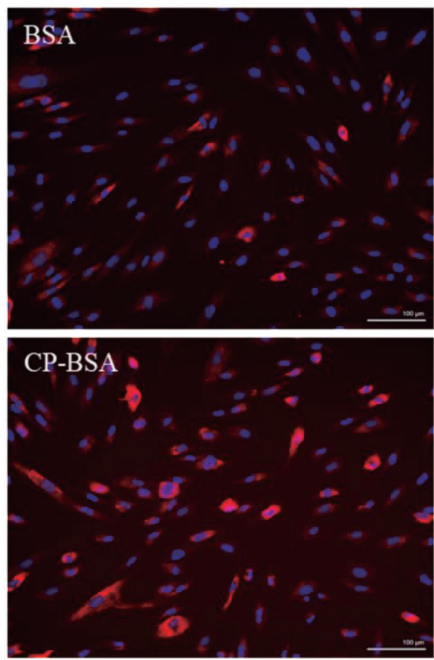

b)
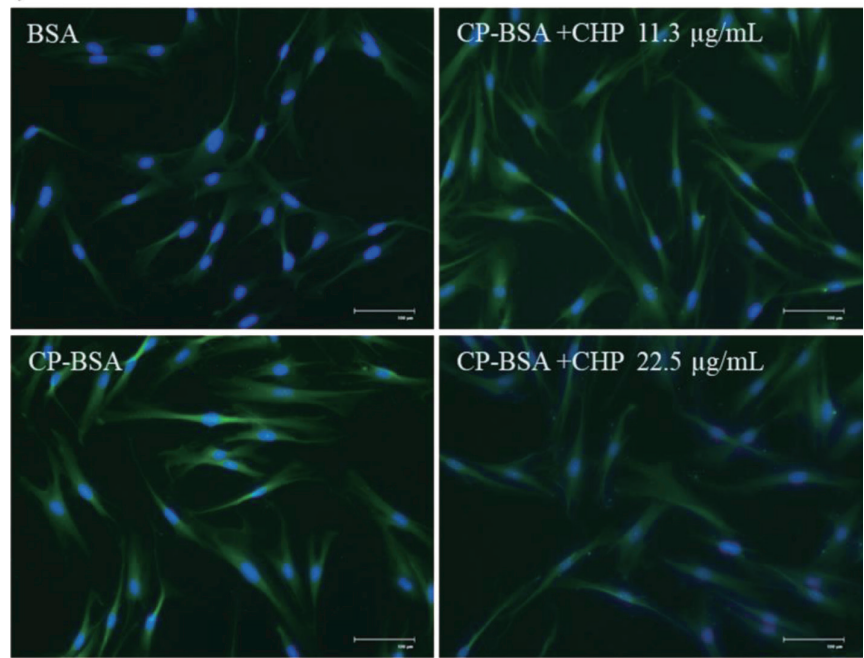

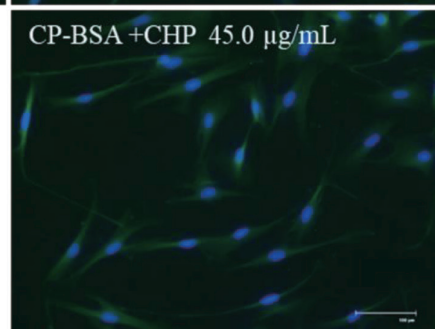

Fig. 1 Acrolein and CPs in fibroblasts treated with CP-BSA and the suppressive effects of CHP on intracellular CP levels. a) At $2 \mathrm{~h}$ after treatment with CP-BSA or BSA, intracellular acrolein in NHDFs was visualized by staining with Acrolein RED. Representative images in triplicate independent examinations are shown. b) At $24 \mathrm{~h}$ after cultivating NHDFs reinoculated after treatment with CP-BSA or BSA in the presence or absence of CHP, intracellular CPs in NHDFs were visualized by fluorescence labeling with FTSC. Representative images in triplicate independent examinations are shown. Scale bars $=100 \mu \mathrm{m}$. 

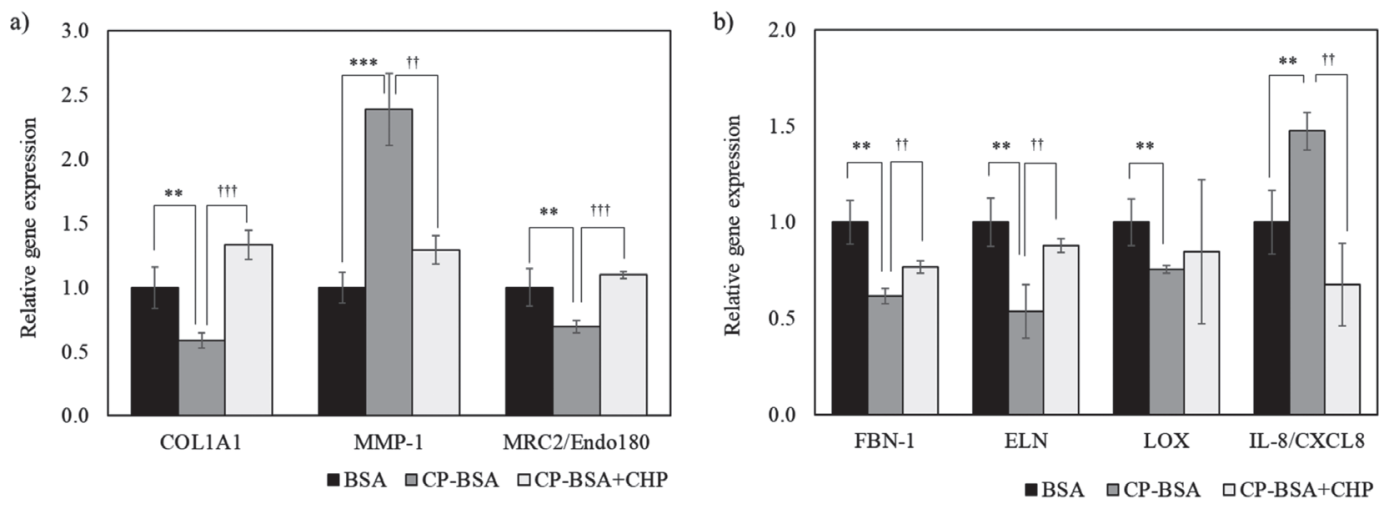

Fig. 2 Restoration effects of CHP on mRNA expression levels of fiber-related proteins altered by CP-BSA.

At $24 \mathrm{~h}$ after cultivating NHDFs reinoculated after treatment with CP-BSA or BSA in the presence or absence of CHP, total RNAs of cells were extracted using an RNeasy Mini Kit. Reverse transcription was performed using the GeneAmp PCR system with ReverTra Ace qPCR RT Master Mix. qRT-PCR was performed using a StepOne ${ }^{\mathrm{TM}}$ system PowerUp $^{\mathrm{TM}}$ SYBR $^{\mathrm{TM}}$ Green Master Mix. GAPDH was used as a housekeeping gene. a) mRNA levels of collagen fiberrelated proteins, b) mRNA levels of elastin fiber-related proteins. Significance: ${ }^{*} p<0.05,{ }^{* *} p<0.01,{ }^{* * *} p<0.001$ (vs. BSA), ${ }^{\dagger \dagger} p<0.01,{ }^{\dagger \dagger} p<0.001$ (vs. CP-BSA).

a)

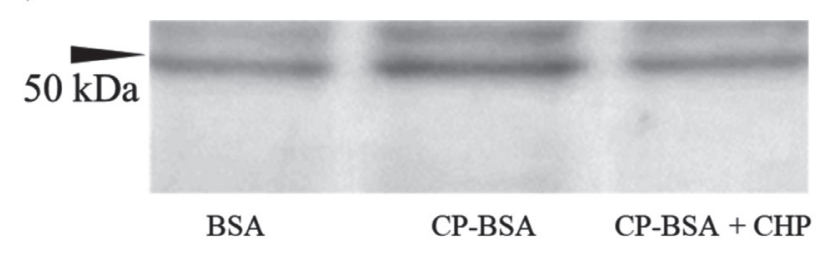

b)

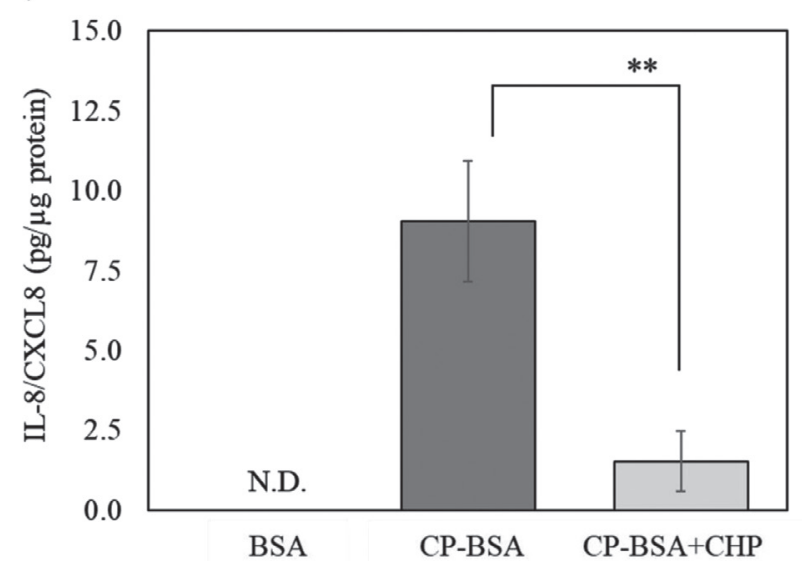

Fig. 3 Restoration effects of CHP on protein expression levels of MMP-1 and IL-8/CXCL8 altered by CPBSA.

At $24 \mathrm{~h}$ after cultivating NHDFs reinoculated after treatment with CP-BSA or BSA in the presence or absence of CHP, the levels of MMP-1 (a) and IL-8/ CXCL8 (b) proteins in the culture medium were analyzed using Western blotting and ELISA, respectively. Significance: $* * p<0.01$.

\subsection{Improving effects of CHP on the formation of collagen and elastic fibers}

Collagen and elastin fibers are assembled by the integrated actions of dermal matrix related proteins. Thus, the influence of CP-BSA on the formation of those fibers and the effects of CHP to improve the formation of both types of fibers disrupted by CP-BSA were examined. Although treatment with CP-BSA disrupted the formation of collagen fibers and microfibrils, treatment with CHP restored their formation (Fig. 4).

\section{Discussion}

In chronically sun-exposed skin, CPs are a footprint of oxidative stress and are present at a higher frequency in the dermis ${ }^{1)}$. The cellular functions of fibroblasts can be modulated by surrounding CPs and may cause some problems in the metabolism of the dermal matrix. In fact, our previous study suggested the possibility that CP-BSA, which is a model of extracellular soluble CPs, promotes the degradation of dermal matrix proteins by MMP-1 and IL-8/ CXCL8 that are upregulated at the mRNA expression level ${ }^{6)}$. MMP-1 is a critical enzyme that cleaves fibrillar type I collagen, and IL-8/CXCL8 is an important chemokine that recruits neutrophils that produce elastase in the dermis ${ }^{10-13)}$. Therefore, it is presumed that increases in the levels of those proteins in the dermis would cause the dermal matrix to have a fragile structure due to the degradation of collagen and elastin fibers. Because a typical feature of the photodamaged dermal structure is the depletion of collagen fibers and the disappearance of fine elastin fibers showing a candle stick-like structure in the papillary region $^{14-16)}$, the presence of extracellular soluble CPs in 
Type I collagen
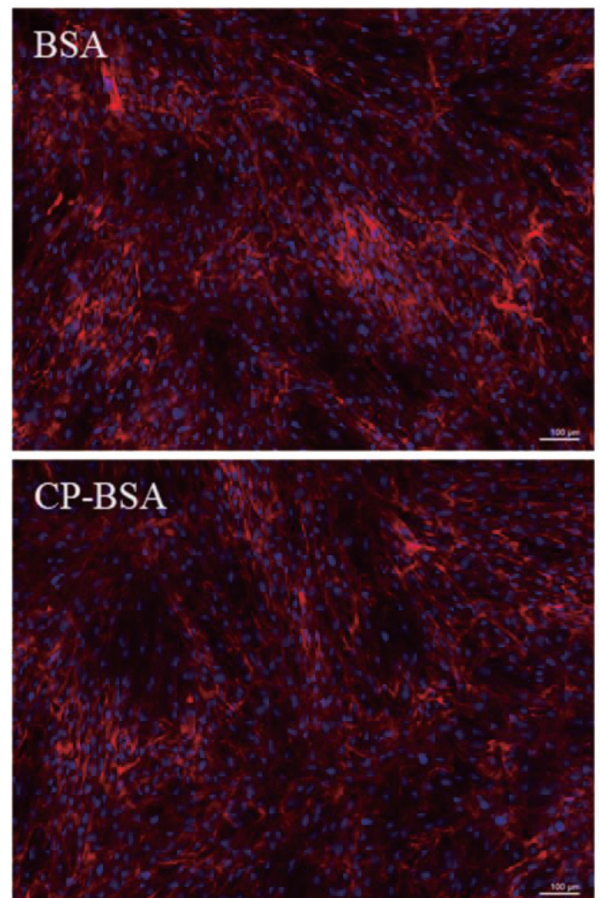

CP-BSA +CHP $45.0 \mu \mathrm{g} / \mathrm{mL}$
Fibrillin-1

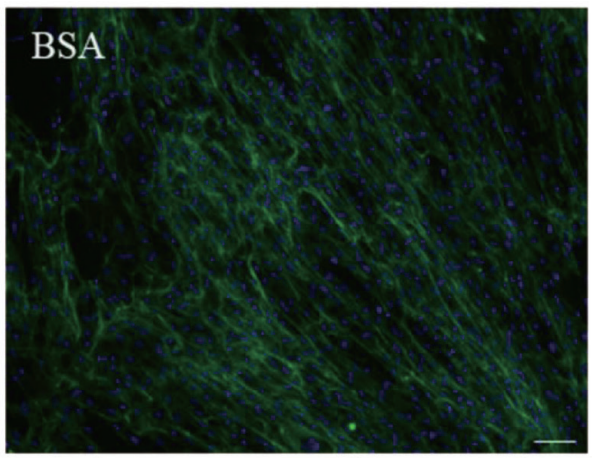

CP-BSA

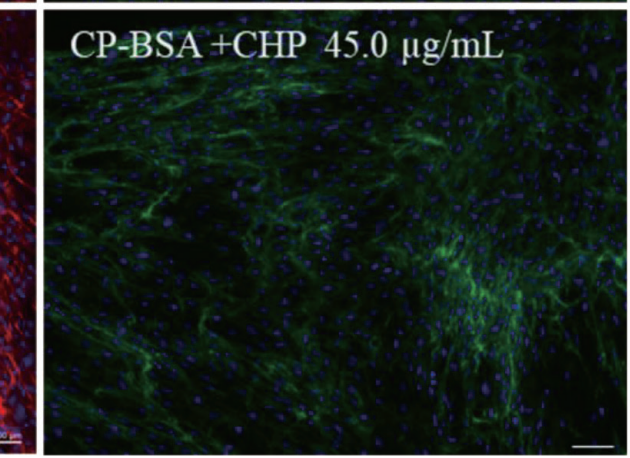

Fig. 4 Restoration effects of CHP on the formation of collagen and elastin fibers impaired by CP-BSA.

NHDFs were cultured with CP-BSA or BSA in the presence or absence of CHP for 1 week; type I collagen fibers were visualized by immunofluorescence staining with an anti-type I collagen antibody (red, left column). Elastic fibers, microfibrils, were visualized by immuno-fluorescence staining with an anti-fibrillin-1 antibody (green, right column). Nuclei were stained with Hoechst 33342 (blue). Representative images in triplicate independent examinations are shown. Scale bars $=100 \mu \mathrm{m}$.

the dermis is expected to promote the progression of photoaged skin even in situations without exposure to the sun. Thus, this study was conducted to clarify the potential improvement effects of xanthophyll carotenoid analogs derived from $\mathrm{HP}$ (CHP) on the formation of collagen and elastin fibers impaired by extracellular soluble CPs associated with the expression of dermal matrix fiber-related proteins.

Oxidative stress initiated by ROS has been shown to be a major inducer of the depletion of collagen and elastin fibers in the dermis, which is a typical feature of photoaged dermis ${ }^{17}$. Furthermore, ROS has been shown to trigger the expression of MMP-1 and IL-8/CXCL8 in an in-vitro study ${ }^{18-20)}$. These facts strongly indicate the relationship between ROS levels and the dysfunction of fibroblasts to maintain the dermal structure. On the other hand, the synthesis of CPs, which are also termed lipid-peroxidation end-products, is initiated by aldehyde compounds yielded by lipid peroxidation by ROS $^{21}$. In a previous study, we found that CP-BSA elevates intracellular levels of ROS in fibroblasts, and results in the accumulation of CPs in fibroblasts ${ }^{6}$. The process of CP accumulation is considered to result in the generation of aldehyde compounds such as acrolein. In fact, our study showed the generation of acrolein in fibroblasts exposed to CP-BSA (Fig. 1a). Gathering these facts, extracellular soluble CPs, such as CP-BSA, might produce new intracel- 
lular CPs through the ROS-acrolein pathway. Thus, in the evaluation of CHP, it is important to first examine whether CHP reduces levels of intracellular oxidative stress that were elevated by CP-BSA through the reduction of acrolein. Xanthophyll carotenoids such as astaxanthin are wellknown quenchers of singlet oxygen ${ }^{22,23)}$. Furthermore, it has been reported that carotenoids exhibit scavenging capabilities against other types of ROS such as $\mathrm{H}_{2} \mathrm{O}_{2}{ }^{24)}$. Indeed, CHP exhibited a scavenging ability against acrolein, and also reduced intracellular CP levels that were elevated by CP-BSA (Table 1, Fig. 1). Currently, it is difficult to formulate a hypothesis about the mechanism involved in the scavenging of acrolein by CHP, because it is hard to explain how CHP, which includes compounds with conjugated double bonds, reacts with $\alpha, \beta$-unsaturated aldehydes like acrolein from a chemical aspect. In thinking about the reduction of levels of intracellular CPs by CHP, it is reasonable to understand the results that CHP scavenged intracellular ROS. However, since it is true that CHP scavenges acrolein, it might be impossible to rule out that CHP suppresses the elevation of intracellular CPs induced by CP-BSA through the scavenging of intracellular acrolein. Thus, we will continue this study to identify the scavenging mechanism of CHP against acrolein and will report that elsewhere.

Summarizing these results, it was expected that CHP would restore the dysfunction of fibroblasts to improve the dermal structure impaired by CP-BSA due to its reduction of intracellular oxidative stress. Thus, we examined the effects of CP-BSA on mRNA and protein expression levels of collagen and elastin fiber-related proteins and evaluated the restoration effects of CHP on alterations caused by CPBSA.

Treatment with CP-BSA downregulated the mRNA expression levels of COL1A1, MRC2, FBN-1, ELN and LOX but upregulated MMP-1 and IL-8/CXCL8 (Fig. 2). Those alterations of mRNA expression levels were presumed to influence the formation of collagen and elastin fibers, and to affect the physiology of fibroblasts as follows: Since COL1A1, FBN-1 and ELN are structural proteins of collagen or elastin fibers, the downregulation of their mRNA levels indicates that the production of those fibers will be reduced. LOX is a crosslinking enzyme that assembles strong fibers of collagen and elastin ${ }^{25-27)}$. In fact, fibroblasts treated with CP-BSA showed a reduced formation of both types of fibers (Fig. 4). Regarding MMP-1 and IL-8/CXCL8, increases in their levels in the dermis would result in a more fragile dermal matrix due to the degradation of collagen and elastic fibers as described above. MRC2, which is localized at the cell membrane of fibroblasts, is a receptor for collagen and internalizes denatured collagen in order to utilize its amino acids for the new synthesis of collagen peptides $^{28)}$. The downregulation of MRC2 suggests an impaired function to clean up denatured collagen at the pe- riphery of fibroblasts and a reduced capacity to supply amino acids for the synthesis of new collagen. Namely, the loss of MRC2 expression suggests that denatured collagen such as carbonylated collagen peptides will remain for a long time in the dermis and may enhance concerns about the influence of carbonylated collagen on the cellular functions of fibroblasts.

In contrast to these alterations of fiber-related proteins caused by CP-BSA, CHP improved their mRNA expression levels, except for LOX, and also restored protein expression levels of MMP-1 and IL-8/CXCL8(Figs. 2 and 3). Although the reason why CHP failed to restore the mRNA expression level of LOX is unclear, oxidative stress or ROS might not be a major regulator of LOX expression. Finally, CHP improved the formation of structural fibers impaired by CP-BSA (Fig. 4). In this study, microfibrils were detected as elastin fibers by immunostaining with an anti-FBN-1 antibody, because fine elastin fibers, which disappear in photoaged dermis, are recognized as oxytalan fibers, in which microfibrils are a major component ${ }^{29)}$.

Taken together, the effects of CP-BSA on the expression of collagen and elastin fiber-related proteins and the improvement effects of CHP against the influence of CP-BSA lead us to conclude the following: Extracellular soluble CPs, which are synthesized by protein oxidation with aldehyde compounds like acrolein, increase intracellular levels of CPs in fibroblasts and suppress the formation of collagen and elastin fibers associated with alterations of fiber-related proteins at the mRNA and protein expression levels. Although it is currently unclear whether acrolein or increased levels of intracellular CPs are the major cause of these phenomena triggered by extracellular soluble CPs, treatment with CHP, which scavenges acrolein and reduces levels of intracellular CPs, resulted in the apparent improvement of these phenomena.

Thus, we conclude that CHP is an effective natural xanthophyll carotenoid that can have anti-aging effects due to its improvement of the dysformation of collagen and elastic fibers caused by extracellular soluble CPs that accumulate in the dermis at sun-exposed sites.

\section{References}

1) Ogura, Y.; Kuwahara, T.; Akiyama, M.; Tajima, S.; Hattori, K.; Okamoto, K.; Okawa, S.; Yamada, Y.; Tagami, H.; Takahashi, M.; Hirao, T. Dermal carbonyl modification is related to the yellowish color change of photoaged Japanese facial skin. J. Dermatol. Sci. 64, 45-52 (2011).

2) Ohshima, H.; Oyobikawa, M.; Tada, A.; Maeda, T.; Takiwaki, H.; Itoh, M.; Kanto, H. Melanin and facial skin fluorescence as markers of yellowish discoloration with aging. Skin Res. Technol. 15, 496-502 (2009). 


\section{Y. Yamawaki, T. Mizutani, Y. Okano et al.}

3) Masaki, H.; Mizutani, T.; Ogawa, N.; Suzuki, H.; Okano, Y.; Akita, H. Carbonylated proteins contribute to the darkness around facial pores. J. Dermatol. Sci. 89, 299-307 (2018).

4) Iwai, I.; Shimadzu, K.; Kobayashi, Y.; Hirao, T.; Etou, T. Increased carbonyl protein level in the stratum corneum of inflammatory skin disorders: A non-invasive approach. J. Dermatol. 37, 693-698(2010).

5) Mizutani, T.; Sumida, H.; Sagawa, Y.; Okano, Y.; Masaki, H. Carbonylated proteins exposed to UVA and to blue light generate reactive oxygen species through a type I photosensitizing reaction. J. Dermatol. Sci. 84, 314321 (2016).

6) Yamawaki, Y.; Mizutani, T.; Okano, Y.; Masaki, H. The impact of carbonylated proteins on the skin and potential agents to block their effects. Exp. Dermatol. 28, 32-37 (2019).

7) Aldini, G.; Orioli, M.; Carini, M. $\alpha, \beta$-Unsaturated aldehydes adducts to actin and albumin as potential biomarkers of carbonylation damage. Redox Report 12, 20-25 (2007).

8) Hirao, T.; Takahashi, M. Carbonylation of cornified envelopes in the stratum corneum. FEBS Letters 579, 6870-6874(2005).

9) Pradipta, A.R.; Taichi, M.; Nakase, I.; Saigitbatalova, E.; Kurbangalieva, A.; Kitazume, S.; Taniguchie, N.; Tanaka, K. Uncatalyzed click reaction between phenyl azides and acrolein: 4-formyl-1,2,3-triazolines as "Clicked" markers for visualizations of extracellular acrolein released from oxidatively stressed cells. ACS Sensors 1, 623-632 (2016).

10) Page-McCaw, A.; Ewald, A.J.; Werb, Z. Matrix metalloproteinases and the regulation of tissue remodelling. Nat. Rev. Mol. Cell Biol. 8, 221-233(2007).

11) Gross, J.; Lapiere, C.M. Collagenolytic activity in amphibian tissues: A tissue culture assay. Proc. Natl. Acad. Sci USA 48, 1014-1022 (1962).

12) Stamenkovix, I. Extracellular matrix remodelling: The role of matrix metalloproteinases. J. Pathol. 200, 448464 (2003).

13) Harada, A.; Sekido, N.; Akahoshi, T.; Wada, T.; Mukaida, N.; Matsushima, K. Essential involvement of interleukin-8 (IL-8) in acute inflammation. J. Leukocyte Biol. 56, 559-564 (1994).

14) Watson, R.E.; Griffiths, C.E.; Craven, N.M.; Shuttleworth, C.A.; Kielty, C.M. Fibrillin-rich microfibrils are reduced in photoaged skin. Distribution at the dermalepidermal junction. J. Invest. Dermatol. 112, 782-787 (1999).

15) Schwartz, E.; Cruickshank, F.A.; Christensen, C.C.; Perlish, J.S.; Lebwohl, M. Collagen alterations in chronically sun-damaged human skin. Photochem. Photobiol. 58, 841-844(1993).

16) Smith, J.G. Jr.; Davidson, E.A.; Sams, W.M.Jr.; Clark,
R.D. Alterations in human dermal connective tissue with age and chronic sun damage. J. Invest. Dermatol. 39, 347-350 (1962).

17) Wlaschek, M.; Tantcheva-Poór, I.; Naderi, L.; Ma, W.; Schneider, L.A.; Razi-Wolf, Z.; Schüller, J.; Scharffetter-Kochanek, K. Solar UV irradiation and dermal photoaging. J. Photochem. Photobiol. B: Biolog. 63 (1-3), 41-51 (2001).

18) Brenneisen, P.P.; Wenk, J.; Klotz, L.O.; Wlaschek, M.; Briviba, K.; Krieg, T.; Sies, H.; Scharffetter-Kochanek, $\mathrm{K}$. Central role of ferrous/ferric iron in the ultraviolet $\mathrm{B}$ irradiation-mediated signaling pathway leading to increased interstitial collagenase (matrix-degrading metalloprotease (MMP) -1) and stromelysin-1 (MMP-3) mRNA levels in cultured human dermal fibroblasts. $J$. Biol. Chem. 273, 5279-5287(1998).

19) Ryan, K.A.; Smith, M.F. Jr.; Sanders, M.K.; Ernst, P.S. Reactive oxygen and nitrogen species differentially regulate toll-like receptor 4-mediated activation of NF-B and interleukin-8 expression. Infect. Immun. 72, 2123-2130 (2004).

20) Fisher, G.J.; Datta, S.C.; Talwar, H.S.; Wang, Z.Q.; Varani, J.; Kang, S.; Voorhees, J.J. Molecular basis of suninduced premature skin ageing and retinoid antagonism. Nature 379, 335-339 (1996).

21) Vistoli, G.; De Maddis, D.; Cipak, A.; Zarkovic, N.; Carini, M.; Aldini, G. Advanced glycoxidation and lipoxidation end products (AGEs and ALEs): an overview of their mechanisms of formation. Free Radic. Res. 47, 3-27 (2013).

22) Terao, J. Production and quenching of singlet molecular oxygen - Its role in oxidative stress -. Vitamins 90, 525-536 (2016).

23) Foote, C.S.; Chang, Y.C.; Denny, R.W. Chemistry of singlet oxygen. X. Carotenoid quenching parallels biological protection. J. Am. Chem. Soc. 92, 5216-5218 (1970).

24) Nakajima, Y.; Shimazawa, M.; Otsubo, K.; Ishibashi, T.; Hara, H. Zeaxanthin, a retinal carotenoid, protects retinal cells against oxidative stress. Curr. Eye Res. 34, 311-318 (2009).

25) Pinnell, S.R.; Martin, G.R. The cross-linking of collagen and elastin: enzymatic conversion of lysine in peptide linkage to alpha-aminoadipic-delta-semialdehyde (allysine) by an extract from bone. Proc. Natl. Acad. Sci. USA 61, 708-716 (1968).

26) Hornstra, I.K.; Birge, S.; Starcher, B.; Bailey, A.J.; Mecham, R.P.; Shapiro, S.D. Lysyl oxidase is required for vascular and diaphragmatic development in mice. $J$. Biol. Chem. 278, 14387-14393(2003).

27) Seve, S.; Decitre, M.; Gleyzal, C.; Farjanel, J.; Sergeant, A.; Ricard-Blum, S.; Sommer, P. Expression analysis of recombinant lysyl oxidase (LOX) in myofibroblastlike cells. Connect. Tissue Res. 43, 613-619(2002). 
28） Tang, S.; Lucius, R.; Wenck, H.; Gallinat, S.; Weise, J.M. UV-mediated downregulation of the endocytic collagen receptor, Endo180, contributes to accumulation of extracellular collagen fragments in photoaged skin. $J$.
Dermatol Sci. 70, 42-48(2013).

29) Cotta-Pereira, G.; Guerra, R.F.; Bittencourt-Sampaio, S. Oxytalan, elaunin, and elastic fibers in the human skin. J. Invest. Dermatol. 66, 143-148(1976). 